\title{
GFP/HPV-16E6 fusion protein induces apoptosis in MCF-7 and 293T cells using a transient expression system
}

\author{
GE ZHANG ${ }^{1 *}$, YAN LIU ${ }^{2 *}$, LIWEI YU ${ }^{1}$ and LINA SUN ${ }^{3}$ \\ ${ }^{1}$ Beijing Haidian Hospital, Beijing 100080; ${ }^{2}$ Beijing Obstetrics and Gynecology Hospital, Capital Medical University, \\ Beijing 100026; ${ }^{3}$ State Key Laboratory for Infectious Disease Prevention and Control, National Institute for Infectious \\ Disease Control and Prevention, Chinese Center for Disease Control and Prevention, Beijing 102206, P.R. China
}

Received June 6, 2012; Accepted July 17, 2012

DOI: $10.3892 / o r .2012 .1976$

\begin{abstract}
Since mucosal high-risk human papillomavirus (HPV) E6 can target and degrade the tumor suppressor p53, it is recognized as a major causative agent of cervical cancer. However, to date the distribution of high-risk HPV-E6 protein remains elusive. Thus, in the present study we used a mammalian green fluorescent protein (GFP) expression system to express a GFP/HPV-16E6 fusion protein (GFP-16E6) in wildtype (wt) p53 cells, such as MCF-7 and 293T cells to investigate the trafficking and localization of E6 and p53. Following transfection, we observed that the overexpressed GFP-16E6 was a nuclear protein, and that endogenous wt p53 localized to the nucleus together with GFP-16E6. Strikingly, p53 levels were not decreased but increased in $24 \mathrm{~h}$ transfected with pGFP16E6. Furthermore, we observed significant apoptosis induced by GFP-16E6, which proved to be dependent on p53 expression.
\end{abstract}

\section{Introduction}

Human papillomaviruses (HPVs) are small double-stranded DNA viruses with a genome of $\sim 8 \mathrm{kB}$. Over $90 \%$ of human cervical carcinoma has been shown to be associated with high risk HPVs, mainly the serotypes 16 and 18 (1). The mechanisms underlying the carcinogenesis of high risk HPVs have been studied extensively, showing that the E6 and E7 proteins are

Correspondence to: Professor Liwei Yu, Department of General Surgery, Beijing Haidian Hospital, 29 Zhongguancun Road, Beijing 100080, P.R. China

E-mail:yu.liwei@yahoo.cn

Professor Lina Sun, State Key Laboratory for Infectious Disease Prevention and Control, National Institute for Infectious Disease Control and Prevention, Chinese Center for Disease Control and Prevention, Beijing 102206, P.R. China

E-mail: sunlina_77@yahoo.com

*Contributed equally

Key words: human papillomavirus, high risk HPV-E6, green fluorescent protein, endogenous wild-type p53, apoptosis the oncoproteins, which interact respectively with essential components of the cellular regulatory machinery, leading to the dysregulated proliferation and transformation of the infected cells (2). The viral E6 protein's principal target is cellular tumor suppressor p53, as a consequence of this interaction, p53 is labeled with ubiquitin, leading to p53 entering ubiquitin-mediated degradation system, therefore, the p53 growth regulatory function is abolished (3). Thus, it has been well accepted that HPV-E6 targeted p53 degradation resulting in p53 pathway failure, together with E7 protein interacted with $\mathrm{pRb}$, is responsible for carcinogenesis $(4,5)$.

p53 is a very important tumor suppressor protein, it remains at low levels under normal conditions, only in response to stress, such as UV radiation, DNA damage, hypoxia or virus infection, p53 gene starts to be activated and the protein expressed (6-8). Activation of $\mathrm{p} 53$ can be modulated at different levels: increased p53 expression, transformation of the protein from a latent to an active conformation through different mechanisms, such as post-translational modification, and translocation of p53 to the nucleus, where it acts as a transcriptional factor $(9,10)$. Little is known about whether the overexpression of high risk HPV-E6 proteins alters the expression and location of endogenous wildtype (wt) p53 protein, and what happens next.

The traffic and distribution of E6 protein inside the infected cells remains elusive. Some authors have shown that the full-length high risk HPV-E6 was located in the nuclei in transiently transfected COS cells by immunofluorescence staining and considered it a nuclear protein (11). Some other studies found it to have both nuclear and cytoplasmic distribution $(12,13)$. These confusing results were probably due to the lack of reliable anti-HPV-16E6 antibodies, and the risk of introducing artifacts into protein distribution from the fixation procedures (14). We used green fluorescent protein (GFP) as a tag labeling HPV-16E6 (GFP-16E6) to track its subcellular location in living cells to get ride of any artificial interference. In the present experiment, an expression plasmid of GFP with HPV-16E6 inserted was applied to transfect wt p53 cell lines, such as MCF-7 and 293T cells, the experiment system would provide a platform for tracing the E6 protein. Simultaneously, we observed the expression, localization, and traffic of p53 protein with immunofluorescence technique, to determine whether the expression of E6 protein would affect the behavior of p53. By immunoblotting, we studied the 
expression level of p53 in the context of E6. Strikingly, the p53 was not degraded in $24 \mathrm{~h}$ in pGFP-16E6 transfected cells.

We observed the stabilization and increased expression of p53 in the presence of overexpressed E6 proteins clearly in the short-term. To avoid the possible effect of GFP-fusion protein on E6-p53 binding and the degradation of p53, we used His tagged HPV-16E6 protein (His-16E6) at the same time. We observed His-16E6 was mainly located in nuclei together with p53, and the p53 was not degraded in $24 \mathrm{~h}$ in His-16E6 expressing cells. Furthermore, at the later times of transfection, p53 was degraded gradually whereas the other apoptosis associated proteins such as bax, Bak, c-myc and cdc2 were increased and bcl-2 was decreased compared with control. We further observed obvious apoptosis induced by E6, which was proved to be dependent on p53 expression. Taken together, in the transient expression system, the high risk HPV-16E6 was located in nuclei together with endogenous wt p53, which in turn induced apoptosis.

\section{Materials and methods}

Plasmid construction. Full length HPV-16E6 was amplified by PCR from HPV type 16 complete genome, and then cloned in frame within the $\mathrm{C}$ terminus of the mammalian expression vector pEGFP-C1 (Clontech, CA, USA) and pcDNA4/To/mycHisC (Invitrogen, CA, USA) respectively, producing plasmids pGFP-16E6 and pcDNA4/To/myc-HisC-16E6.

Cell culture and transfection. The human breast adenocarcinoma MCF-7 cells and human embryonic 293T kidney cells were maintained in RPMI1640 medium (Gibco) supplemented with $10 \%$ fetal bovine serum (FBS). Human colon carcinoma HCT116 cells and HCT116 p53 $3^{-/}$were maintained in DMEM (Gibco) supplemented with $10 \% \mathrm{FBS}$, at $37^{\circ} \mathrm{C}$ in a humidified atmosphere of $5 \% \mathrm{CO}_{2}$. The MCF-7 and 293T cells were seeded at approximately $30 \%$ confluency on glass coverslips in 12-well cell culture plates. The cells were transiently transfected with plasmid pGFP-16E6 and pGFP overnight using Lipofectamine 2000 transfection reagent (Invitrogen) following the recommendations of the manufacturer. The reagent:DNA ratio was 2:1.

Viable-cell imaging by confocal microscopy. The MCF-7 and $293 \mathrm{~T}$ cells were grown on glass coverslips, transfected, and at $21 \mathrm{~h}$ post-transfection, coverslips were mounted on modified glass slides with $10 \%$ fetal calf serum-containing cell culture medium, and used immediately for imaging. Images of live cells were collected with a Leica confocal microscope (Leica Microsystems, Wetzler, Germany) at a magnification of $\mathrm{x} 400$. Fluorescent images were analyzed using Leica Confocal Software (Leica Microsystems).

Immunocytochemistry. The cells were seeded on glass coverslips at a density of 100,000 or 200,000 cells/well. Following standard procedures, they were transfected with plasmid pGFP-16E6, pGFP, pcDNA4/To/myc-HisC-16E6 and pcDNA4/ To/myc-HisC, respectively. After transfection, the cells were washed with PBS and fixed with $4 \%$ paraformaldehyde for $10 \mathrm{~min}$ at room temperature. They were then rehydrated three times with cold PBS, permeabilized with $1 \%$ Triton X-100 for
5 min on ice, and rinsed with PBS and blocked. The pGFP and pGFP-16E6 transfected cells were incubated with a primary antibody against p53 (cell signaling: dilution, 1:500) overnight at $4^{\circ} \mathrm{C}$. Subsequently, signal detection was performed using Cy3-conjugated goat anti-rabbit IgG (Sigma; dilution, 1:200) in blocking solution for $30 \mathrm{~min}$ at room temperature in the dark. Then, the cells were washed three times with PBS and examined by confocal microscopy.

The pcDNA4/To/myc-HisC and pcDNA4/To/myc-HisC16E6 transfected cells were incubated with a primary antibody against His (Clontech, dilution 1:500) overnight at $4^{\circ} \mathrm{C}$, the secondary antibody of HRP-conjugated goat anti-mouse $\mathrm{IgG}$ (Pierce, Rockford, IL; dilution, 1:500) in blocking solution for $30 \mathrm{~min}$ at room temperature. Reaction products were visualized with 3,3'-diaminobenzidine tetrahydrochloride (DAB) and the slides were counterstained with hematoxylin.

The pcDNA4/To/myc-HisC and pcDNA4/To/myc-HisC$16 \mathrm{E} 6$ transfected cells were also incubated with primary antibodies against p53 (cell signaling; dilution, 1:500) and against His (Clontech, dilution 1:500) overnight at $4^{\circ} \mathrm{C}$, the secondary antibodies of FITC-conjugated goat anti-mouse IgG (Sigma; dilution, 1:200) and Cy3-conjugated goat antirabbit IgG (Sigma; dilution, 1:200) in blocking solution for $30 \mathrm{~min}$ at room temperature in the dark. Then, the cells were washed three times with PBS and examined by fluorescence microscopy.

Co-immunoprecipitation. Cells $\left(10^{7}\right)$ were lysed in RIPA buffer on ice for 20 min followed by $20 \mathrm{~min}$ of centrifugation at $16,000 \mathrm{x} \mathrm{g}$. Cell lysates $0.5 \mathrm{ml}(1 \mathrm{mg}$ of protein) were clarified with $20 \mu 1$ of Protein G PLUS-Agarose (Santa Cruz Biotechnology) for $1 \mathrm{~h}$ at room temperature. After centrifugation supernatants were incubated with $2 \mu \mathrm{g}$ of anti-p53 rabbit antibody overnight at $4^{\circ} \mathrm{C}$. Protein G-PLUS Agarose $(20 \mu \mathrm{l})$ was then added to cell lysates and incubated for $2 \mathrm{~h}$ at room temperature. Beads were washed three times with PBS and proteins were eluted by the addition of $40 \mu \mathrm{l} 1 \mathrm{X}$ electrophoresis sample buffer. Western blots were performed using anti-GFP mouse antibody (Clontech, JL, CA).

Immunoblotting analysis. For each sample, $10^{6}$ cells were collected by centrifugation (1000 rpm for $5 \mathrm{~min}$ ), washed once with ice cold PBS, and lysed in $100 \mu$ l RIPA buffer containing $50 \mathrm{mM}$ Tris- $\mathrm{HCl}$ (pH 7.4), $150 \mathrm{mM} \mathrm{NaCl}, 1 \% \mathrm{NP}-40,0.5 \%$ sodium deoxycholate, $1 \mathrm{mM}$ EDTA, $2.5 \mathrm{mM}$ glycerophosphate, $1 \mathrm{mM}$ PMSF, $10 \mathrm{mM} \mathrm{NaF}$, and protease inhibitors (Complete Mini, Roche Diagnostics, Mannheim, Germany). Protein concentration was determined using the BCA reagents (Pierce). Samples $(30 \mu \mathrm{g})$ were analyzed on $12 \%$ SDS polyacrylamide gels, transferred to PVDF membranes (Invitrogen), and blocked for $1 \mathrm{~h}$ at room temperature with 5\% non-fat milk in TBS buffer [20 mM Tris- $\mathrm{HCl}(\mathrm{pH} 7.5), 0.5 \mathrm{M} \mathrm{NaCl}$ ]. The membranes were then incubated with the primary antibody overnight at $4^{\circ} \mathrm{C}$. After three washes with TBS, the membranes were incubated with the secondary antibody for $30 \mathrm{~min}$ at room temperature. After three additional washes, the proteins were visualized by enhanced chemiluminescence (ECL) (Amersham Pharmacia, Piscataway, NJ, USA). The amounts of proteins were determined by densitometric scanning (Dinco and Rhenium Biological Imaging System BIS 202). 
A
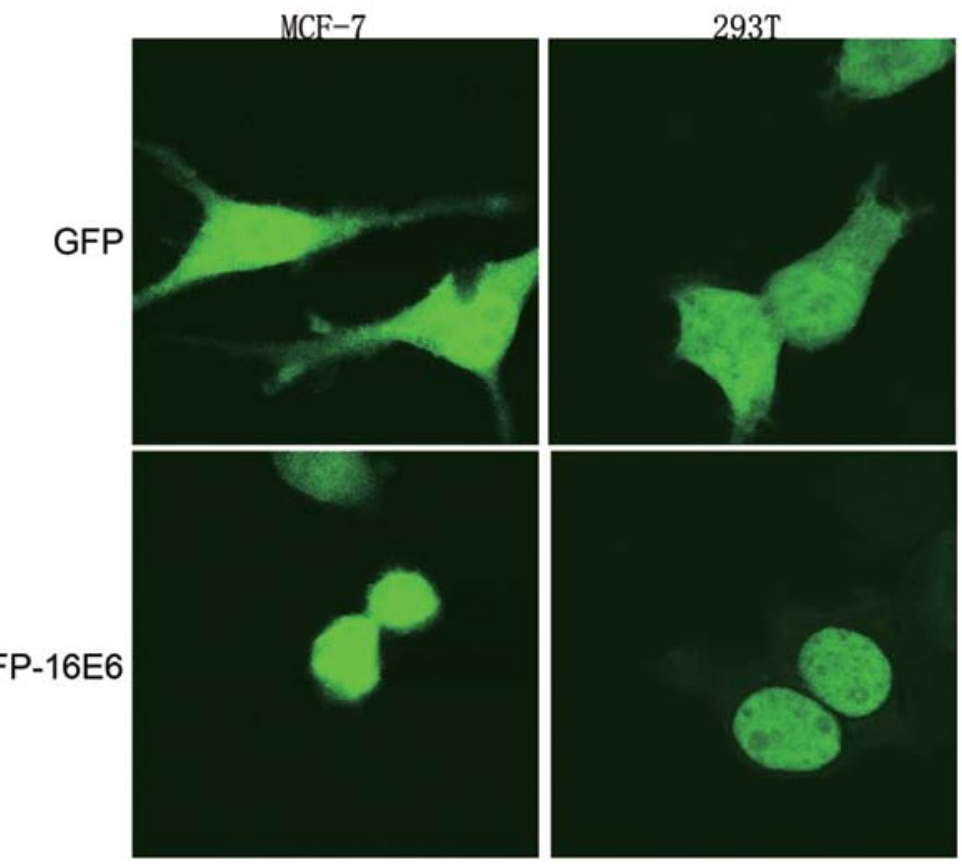

$\mathbf{B}$
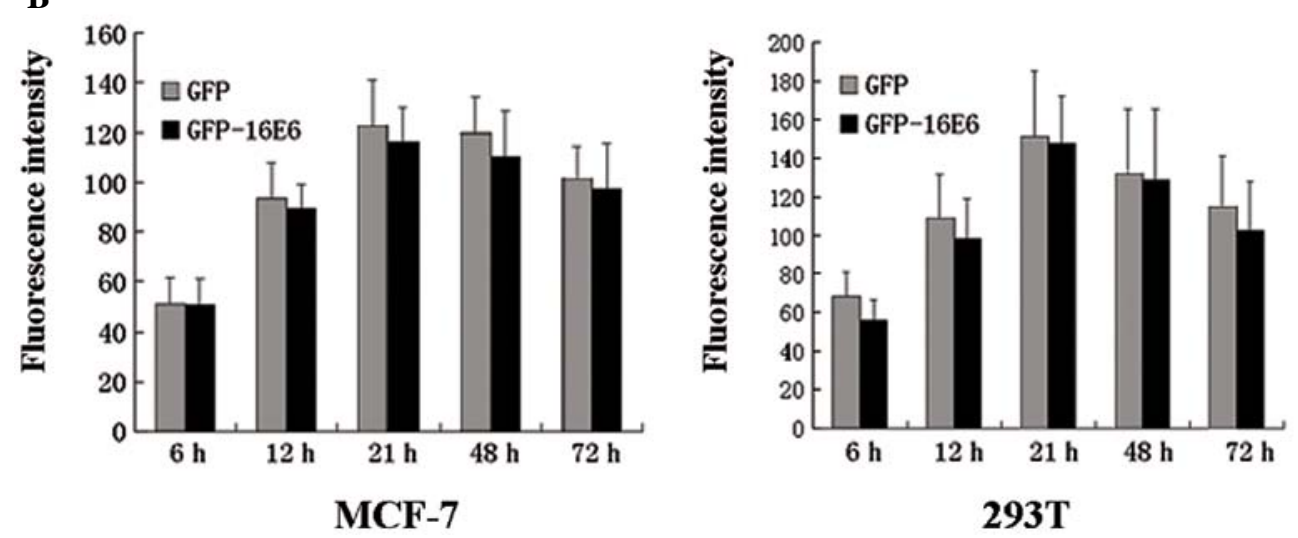

Figure 1. Viable cell images for subcellular localization of GFP and GFP-16E6 proteins in MCF-7 and 293T cells. (A) After $21 \mathrm{~h}$ of transfecting with GFP and GFP-16E6 expression plasmid, the protein GFP is present in both nuclei and cytoplasm, and GFP-16E6 is predominately confined to the nuclei. The results shown are representative of three independent experiments. The green fluorescence is emitted by the cells transfected with pGFP and pGFP-16E6, respectively. (B) The data represent GFP-16E6 and GFP expression from 6 to 72-h post-transfection. The protein expression level is examined by fluorescence intensity. Cells (100) were examined for each plasmid from 20 random fields.

The primary antibodies used were: anti-p53 (Cell Signaling; dilution, 1:1,000), (the following were all from Santa Cruz Biotechnology) anti-bax (dilution 1:500), anti-bcl-2 (dilution 1:500), anti-Bak (dilution 1:500), anti-c myc (dilution 1:500), anti-cdc2 (dilution 1:500) and anti- $\beta$ actin (dilution, 1:10,000). The blots were counterstained with goat anti-mouse or goat anti-rabbit IgG conjugated with HRP (Pierce).

Analysis of apoptosis by flow cytometry using Annexin V and PI double staining. The transfected cells were harvested after 24,48 , and $72 \mathrm{~h}$ by trypsinization, and apoptotic cells were assayed with the Annexin V-APC Apoptosis Detection kit (Bender Medsystems, Burlingame, CA). Briefly, $1 \times 10^{6}$ cells in $100 \mu \mathrm{l}$ binding buffer were stained with $5 \mu \mathrm{l}$ Annexin V-APC and $10 \mu \mathrm{l}$ PI (final concentration, $1 \mu \mathrm{g} / \mathrm{ml}$ ) by mixing and incubating on ice for $10 \mathrm{~min}$ in the dark. The cells were analyzed by flow cytometry. The data were processed using the Cell Quest software.
Statistics. All data were recorded as means \pm standard deviation, and analyzed by the SPSS 11.0 software. Analysis of data was performed using one-way ANOVA for multiple comparisons. P-values $<0.05$ were considered statistically significant.

\section{Results}

GFP-16E6 is a nuclear protein. Viral E6 coding regions were inserted within the $\mathrm{C}$ terminus of the pGFP vector, producing plasmid pGFP-16E6. We transiently transfected pGFP-16E6 in MCF-7 and 293T cells, which allow E6 proteins to be expressed as GFP-16E6 fusion proteins. By confocal microscopy, we observed the subcellular localization of GFP-16E6 and GFP in viable cell images. The E6 fusion proteins may have low or high expression levels at different times, and this could affect the distribution of E6. Therefore, we observed the localization and expression of proteins from 6 to $72 \mathrm{~h}$ post- 

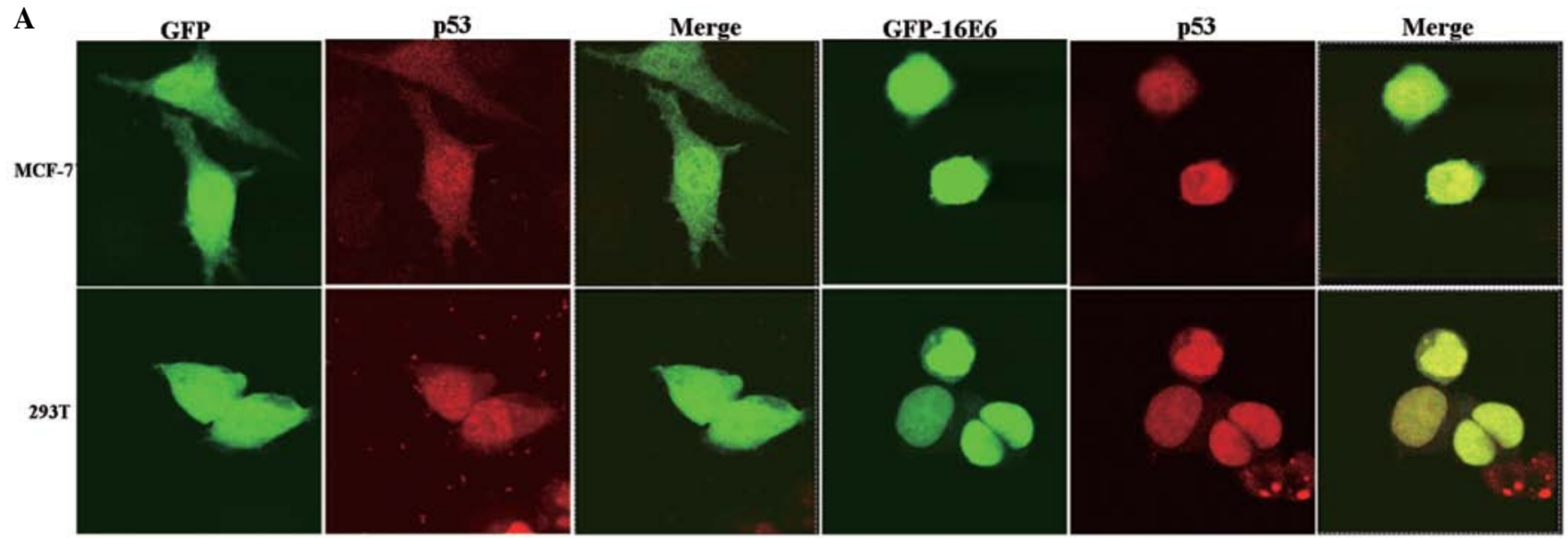

$\mathbf{B}$

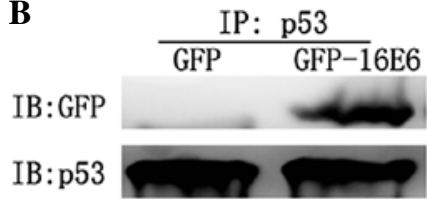

MCF-7

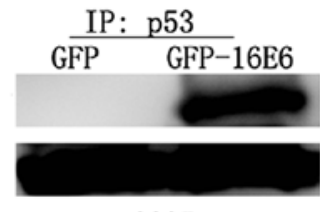

$293 \mathrm{~T}$

Figure 2. Co-localization and interaction of p53 and GFP-16E6 proteins in MCF-7 and 293T cells. (A) The results show that, in pGFP-16E6 transfected cells, both GFP-16E6 and p53 proteins are located in the nuclei. Green fluorescence indicates the protein of GFP and GFP-16E6 expressed by the transfected cells. Red fluorescence indicates 53 protein, which is labeled with anti-P53 antibody plus anti-rabbit-Cy3 secondary antibody. The results shown are representative of three independent experiments. (B) Co-immunoprecipitation of exogenously expressed GFP-16E6 with endogenous p53 protein in MCF7 and $293 \mathrm{~T}$ cells. Lysates from transfected cells expressing GFP-16E6 and GFP alone were submitted to immunoprecipitation with anti-p53 antibodies. Immunoprecipitates were resolved on 12\% SDS-PAGE and immunoblotted with anti-GFP. The results indicate p53 interacted with GFP-16E6 in both MCF-7 and 293T transfected cells. The results shown are representative of three independent experiments.

transfection. The results indicated that GFP-16E6 protein was expressed essentially in the nucleus from $6 \mathrm{~h}$ post-transfection. Its expression increased gradually, and reached its maximum expression level at $21 \mathrm{~h}(\mathrm{P}<0.001)$. Then, it decreased gradually and disappeared after one week. During this whole period, no change was observed in protein localization. As control, we observed the expression of GFP alone. It exhibited a diffused signal, and was present in both the nucleus and cytoplasm from $6 \mathrm{~h}$ to one week post-transfection. In addition, its location did not change at any time (Fig. 1A).

The 293T cells were also used to study E6 localization. The cellular distributions of GFP and GFP-16E6 proteins were similar to those in MCF-7 cells (Fig. 1A). The analysis of relative fluorescence signal intensity of GFP-16E6 in different cell lines is shown in Fig. 1B.

Co-localization of GFP-16E6 and p53 protein. Because high risk E6 can bind to p53 (15), we suspected that the GFP-16E6 and p53 might locate together. Using MCF-7 and 293T cells, we investigated endogenous wt p53 localization by immunocytofluorescence technique. The results showed that $\mathrm{p} 53$ protein was mainly located in the nuclei of pGFP transfected cells. In pGFP-16E6 transfected cells, the distribution of $\mathrm{p} 53$ protein was not changed, and it was located in the nuclei together with GFP-16E6. Fig. 2A shows representative images of the co-localization of GFP-16E6 and p53 proteins.

GFP-16E6 interacts with $p 53$ in vivo. In the present study, we observed p53 and E6 protein located together, it was necessary

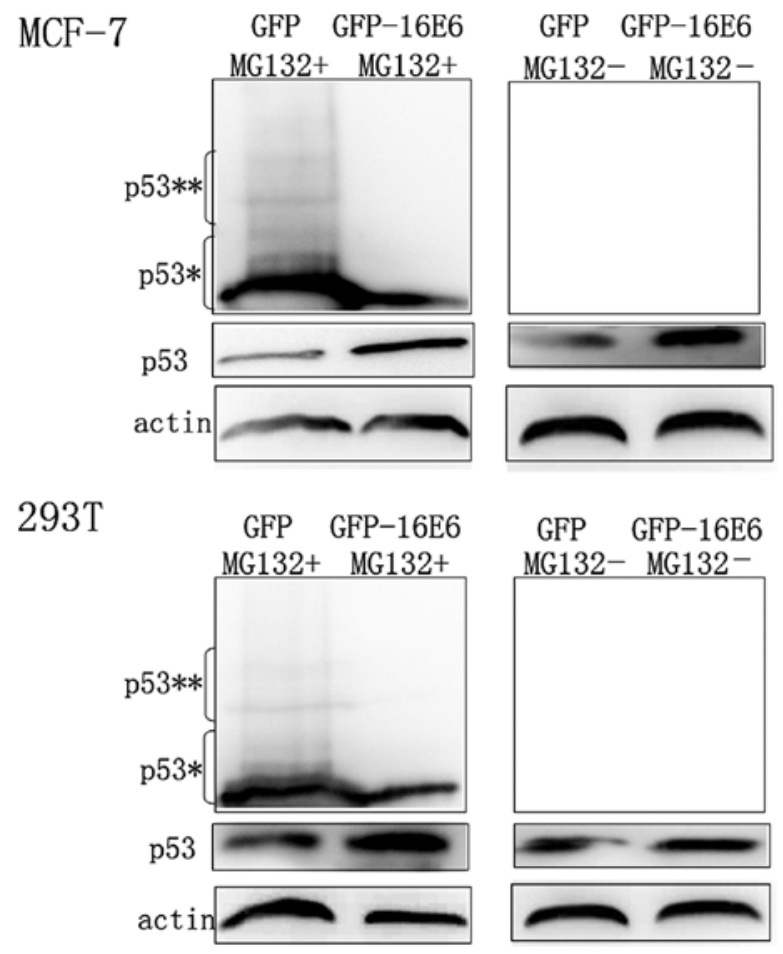

Figure 3. Ubiquitination of p53 is decreased in GFP-16E6 expressing cells. At 24-h post-transfected with pGFP or pGFP-16E6, whole cell lysates are used for immunoblotting with anti-p53 and anti- $\beta$ actin antibodies. As previous reported, the membrane was exposed for a short time (middle panel) to detect the unmodified p53 or for a long time (top panel) to detect the ubiquitinmodified p53 (30). The mono-ubiquitinated and poly-ubiquitinated p53 are marked with $*$ and $* *$, respectively. 
A
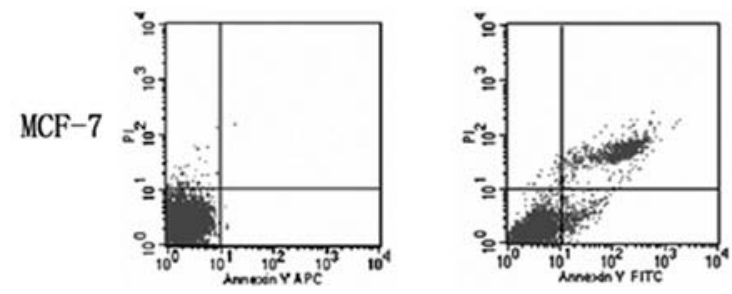

$293 \mathrm{~T}$
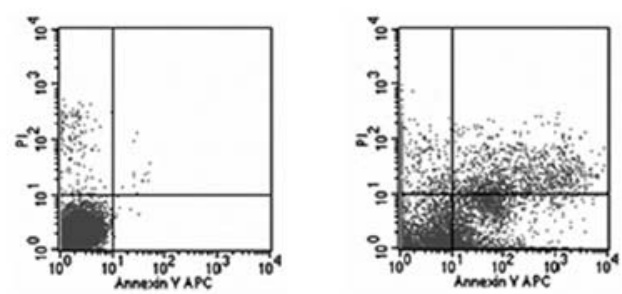
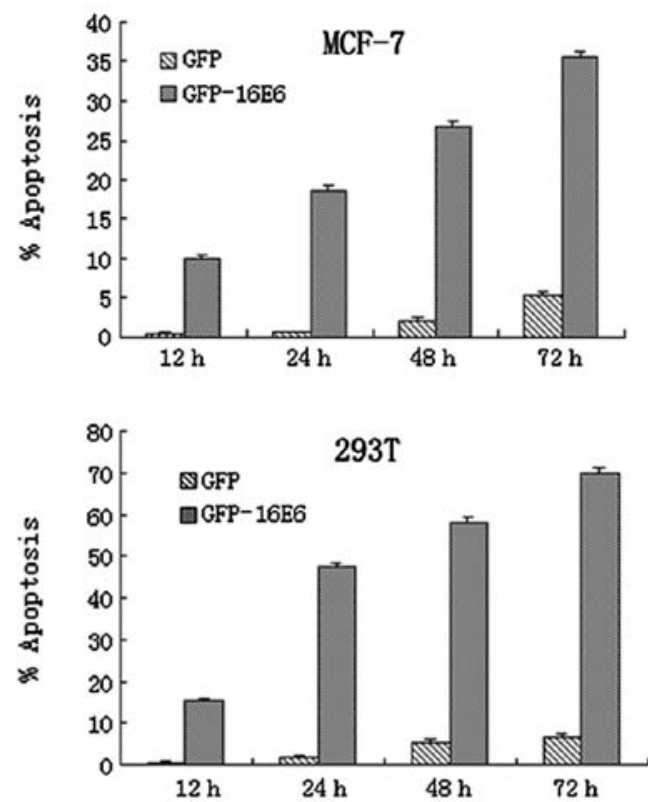
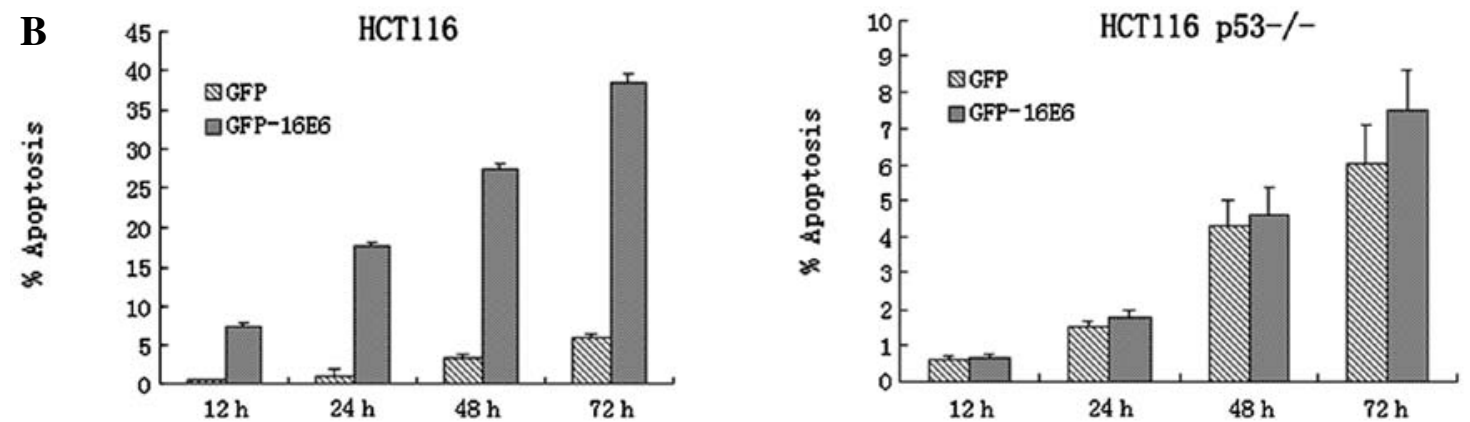

Figure 4. Apoptosis of wt p53 cells transfected with pGFP and pGFP-16E6 analyzed by AnnexinV-PI based flow cytometry assay. Cells were stained with Annexin V and propidium iodide and subjected to cytofluorimetric analysis. Annexin V binding to phosphatidylserine in the absence of propidium iodide staining is indicative of apoptosis in progress. Representative images show apoptosis level at 24-h post-transfection. Results are expressed as percent of apoptotic cell number and data are shown as mean \pm standard deviation of three experiments performed in duplicate. (A) The data of apoptosis induced by HPV-16E6 in MCF-7 and 293T cells from 12 to $72 \mathrm{~h}$ post-transfection. (B) p53 is necessary for GFP-16E6 to induce apoptosis. In the context of E6, there is obvious apoptosis in HCT116 cells, whereas there is no obvious apoptosis in HCT116 p53 $3^{-1-}$ cells.

to determine whether GFP-16E6 could interact with $\mathrm{p} 53$ in vivo. We investigated the potential role of the interaction between endogenous wt p53 and E6 protein by performing immunoprecipitation assay with anti-p53 antibodies. Then, by western blot analyses with anti-GFP antibodies it was shown that p53 interacted with GFP-16E6 protein. Moreover, as a control, in GFP expressing cells, only the GFP protein that lacked the E6 protein was unable to interact with p53 (Fig. 2B). Therefore, in present study, we observed that p53 could interact with HPV-16E6 protein in vivo. This is supported by a report that $\mathrm{p} 53$ has binding sites for high risk HPV-E6 (15).

p53 is increased in $24 \mathrm{~h}$ transfection with $p G F P-16 E 6$. It has been extensively shown that $\mathrm{p} 53$ was degraded by $26 \mathrm{~S}$ proteasome via the ubiquitin pathway (16). Since E6 interaction with p53, we next to determine whether the overexpressed E6 affected this process of $\mathrm{p} 53$ degradation. At $24 \mathrm{~h}$ post-transfection, the GFP and GFP-16E6 expressing cells were treated with MG132, a potent inhibitor of $26 \mathrm{~S}$, and then examined 553 level by immunoblotting. The ubiquitination of $\mathrm{p} 53$ was readily detected in both GFP and GFP-16E6 expressing cells upon MG132 treat- ment. The results showed that the ubiquitination of p53 was significantly lower in GFP-16E6 cells than GFP control cells (Fig. 3). Thus, p53 was increased in $24 \mathrm{~h}$ in GFP-16E6 expressing cells, which was partly due to the decreased ubiquitin-mediated degradation of $\mathrm{p} 53$.

GFP-16E6 induces apoptosis in transfected cells. With the overexpression of oncoprotein E6, we next asked whether it can promote cell apoptosis along with the expression of p53. By Annexin V and PI double-staining combined with flow cytometry, we observed obvious apoptosis in GFP-16E6 expressing MCF-7 cells. Apoptosis occurred at $12 \mathrm{~h}$ post-transfection, and it increased gradually. From 12 to $72 \mathrm{~h}$ post-transfection, apoptosis was prominent compared with GFP alone expressing cells $(\mathrm{P}<0.001)$. For $293 \mathrm{~T}$ cells, we obtained similar result to that in MCF-7 cells (Fig. 4A).

By western blotting, we examined p53 level in GFP-16E6 cells at $12,24,48$ and $72 \mathrm{~h}$ post-transfection. The result indicated, for GFP-16E6 expressed cells, p53 was increased in $24 \mathrm{~h}$ post-transfection, and then it degraded gradually at later times. Accordingly, the other apoptosis associated proteins, 

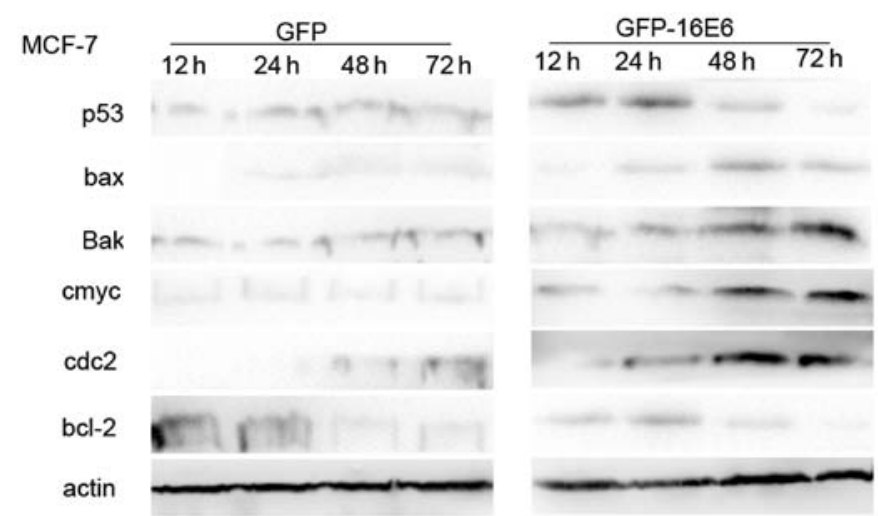

$293 T$

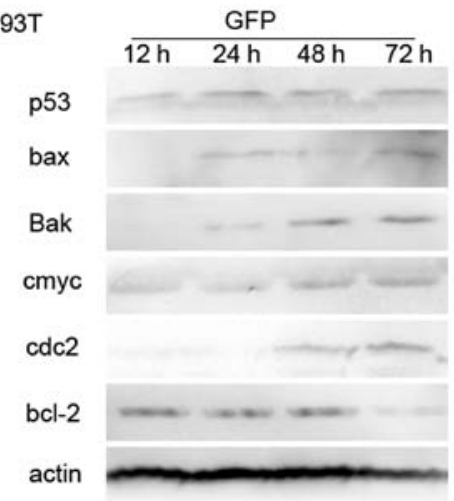

Figure 5. The level of p53 and apoptotic associated proteins in GFP-16E6 expressed in MCF-7 and 293T cells. For both cells, the bands of p53 are increased in $24 \mathrm{~h}$ and then decreased gradually. For p53, bax, Bak, c-myc and cdc2 are increased and bcl-2 is decreased in GFP-16E6 expressing cells compared with GFP control cells at matched times. Data are normalized to $\beta$-actin and representative of three independent western blot analyses.

such as bax, Bak, c-myc and cdc2 were increased and the bcl-2 was decreased compared with GFP control cells (Fig. 5). On the other hand, p53 in GFP-16E6 expressing MCF-7 cells was degraded more than 293T cells. The various activity of E6 to target and degrade p53 was partly due to p53 conformation in different cells (17). Thus, our data indicated the GFP-16E6 could induce apoptosis in wt p53 cell lines.

p53 is necessary for GFP-16E6 induced apoptosis. Our result showed there was obvious apoptosis induced by E6 along with the expression of $\mathrm{p} 53$. To further investigate whether $\mathrm{p} 53$ was necessary for apoptosis, we transfected pGFP-16E6 in both HCT116 and HCT116 p53 ${ }^{--}$cells. We observed, in the context of HPV-16E6, there was obvious apoptosis in HCT116 cells, whereas there was no obvious apoptosis in HCT116 $\mathrm{p} 53^{-/-}$cells (Fig. 4B). Therefore, we concluded p53 was necessary for GFP-16E6 induced apoptosis.

p53 is increased in 24 h by His tagged HPV-16E6 protein expression. To avoid the possible effect of GFP-fusion protein on E6-p53 binding and the degradation of p53, we used His with HPV-16E6 fusion protein for further research. By immunocytochemistry stain, we observed the His-16E6 expression in both MCF-7 and 293T cells (Fig. 6). Furthermore, using immonofluorescent technique, we clearly observed His-16E6 was mainly located in the nuclei together with p53 (Fig. 7A).

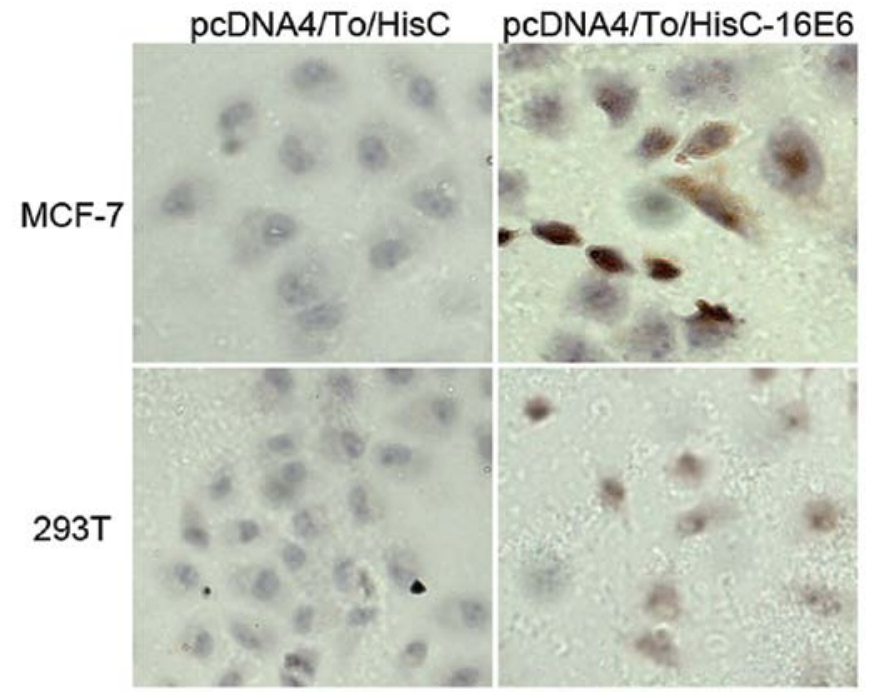

Figure 6. The expression of His-16E6 protein in MCF-7 and 293T cells. The positive stain indicates the expression of His with HPV-16E6 fusion protein, which is labeled with anti-His antibody. The images were examined at $\mathrm{x} 400$ magnification.

Comparing with pcDNA4/To/myc-HisC control cells, the p53 protein expression level was increased in $24 \mathrm{~h}$ along with the His-16E6 expression (Fig. 7B).

\section{Discussion}

The present study provides a novel observation that the transiently expressed high risk HPV-16E6 with GFP fusion protein induced apoptosis in wild-type (wt) p53 cells. We showed HPV-16E6 was a nuclear protein, and the endogenous wt p53 was located in nuclei together with HPV-16E6. Furthermore, there was obvious apoptosis induced by HPV-16E6 which was dependent on $\mathrm{p} 53$ expression.

Many studies on the localization of E6 protein have led to contradictory results, most probably due to the low level of endogenous E6 protein and the poor reactivity of the available anti-E6 antibodies. In the present study, we used GFP with HPV-16E6 fusion proteins to dynamically trace the traffic and localization of E6 proteins in different cell lines. GFP is a convenient, genetically encoded intrinsic fluorescent molecular label that has been widely and successfully used to study protein distribution in cells (18). Our results suggested that GFP-16E6 was mainly expressed in the nucleus of transfected cells. This was consistent with the study by Tao et al who showed that the high risk full-length E6 protein was distributed predominantly in the nucleus of transfected COS-1 cells (14). Thus, it seems likely that the localization of HPV-16E6 in the nucleus is consistent with E6 having some transcription factors (19-21) including p300/CBP, IRF-3, c-Myc or transcriptional co-activators (22) as cellular binding partners which were mainly located in the nuclei.

The tumor suppressor p53 causes cell cycle arrest or apoptosis in response to DNA damage and other forms of stress (23). The ability to localize into the nucleus is essential for p53 to act as a transcription factor. Previous studies have shown the p53 interacting with HPV-E6 playing a very important role in carcinogenesis. We next asked whether the presence of E6 altered 

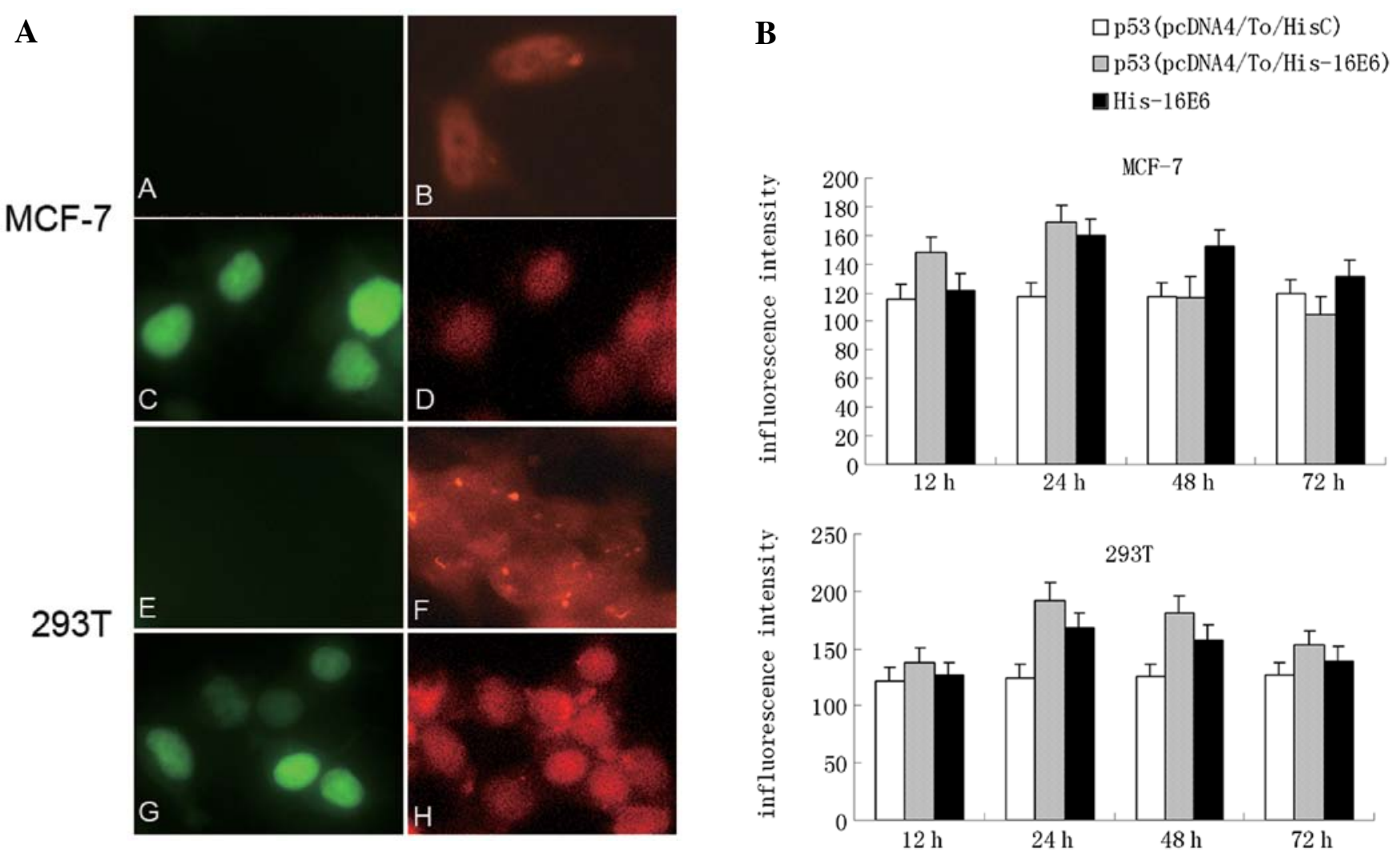

Figure 7. His with HPV-16E6 fusion protein is mainly located in the nuclei together with p53. (A) After $24 \mathrm{~h}$ of transfecting with pcDNA4/To/myc-HisC and pcDNA4/To/myc-HisC-16E6 expression plasmid, the protein His-16E6 was present in the nuclei. In control pcDNA4/To/myc-HisC transfected cells, there was no positive stain (A and E). The green fluorescence ( $C$ and $G$ ) indicates the His-16E6 positive protein. Red fluorescence (B, D, F, and H) indicates p53 protein. The images were examined by fluorescecnt microscopy at $\mathrm{x} 400$ magnification. The results shown are representative of three independent experiments. (B) The protein expression level was examined by fluorescence intensity. One hundred cells were examined for each plasmid from $20 \mathrm{x}$ random fields. p53 is increased in $24 \mathrm{~h}$ in His-16E6 expressing cells and then decreased gradually at later times

the subcellular localization of p53. About $50 \%$ of tumor cells contain mutated p53 but only wt p53 is detected in the HPV sequence positive tumors (24). Therefore, we chose MCF-7 and $293 \mathrm{~T}$ cells, which are wt p53 cell lines, they can partly stimulate HPV-infected cells. We observed the endogenous wt p53 was mainly located in the nuclei together with HPV-16E6, furthermore, we proved E6 interaction with p53 in vivo. These data were consistent with authors who claimed that E6 did not alter the cellular localization of p53, and it was co-localized with p53 (12).

Next, we examined the level of endogenous wt p53 in the context of HPV-16E6. Of note, the result indicated, the endogenous wt p53 was not degraded but increased in $24 \mathrm{~h}$ in GFP-16E6 expressing cells. Also, the same result was obtained several times in both MCF-7 and 293T cells. To avoid the possible effect of GFP-fusion protein on E6-p53 binding and the degradation of p53, we expressed the His with HPV-16E6 fusion protein further for further investigation. We obtained similar result to GFP tagged HPV-16E6. The His-16E6 was mainly located in the nuclei together with p53, and the p53 was increased in $24 \mathrm{~h}$ in His-16E6 expressing cells. This agreed with the GFP tag available for HPV-E6 protein research, and it did not effect the interaction of p53 and E6 $(14,25,26)$. This result was supported by Kawamata et al who reported that $\mathrm{p} 53$ protein expression levels in normal cervical keratinocytes were not degraded by the introduction of HPV-16E6, probably due to a tight transcriptional regulation of p53 (27). This was also supported by increasing evidence, which clearly suggesting that the expression of E6 does not necessarily equate to a p53 null background $(28,29)$.

In present study, we clearly observed p53 was located in the nuclei together with HPV-16E6. Furthermore, we observed in both GFP tag and His tag expressed system, p53 was increased in $24 \mathrm{~h}$ transfected with HPV-16E6. This confirmed that the infected cells recognize virus replication as a DNA damage stress and elicit host surveillance mechanism which ultimately induces activation of p53 (30). We observed obvious apoptosis induced by HPV-16E6 along with the expression of $\mathrm{p} 53$. This finding agreed with the possibility that p53 can transactivate other genes to induce apoptosis in response to the overexpression of E6 (31). Accordingly, our result showed the apoptosis associated proteins, such as bax, Bak, c-myc and cdc2 (18) were upregulated, whereas the bcl-2 was downregulated by HPV-16E6 expression. To investigate whether apoptosis-induced by E6 was dependent on p53 expression, we took advantage of HCT116 and HCT116 p53 $3^{--}$cells, which are a pair of cells: one contain wt p53, the other one is wt p53 null. Also, we proved p53 was necessary for E6 induced apoptosis. It seems likely the activity of p53 is a key event for anti-virus response. There are other viruses, such as EB and Africa Swine Fever virus, they both can induce apoptosis which was dependent on p53 activation $(32,33)$.

In conclusion, we observed that transiently expressed GFP-16E6 was located in the nuclei together with the endogenous 
wt $\mathrm{p} 53$ protein, which in turn induced apoptosis. Therefore, our experiments provided new insight into the interaction of high risk HPV-E6 and endogenous wt p53.

\section{References}

1. Antonsson A, Spurr TP, Chen AC, et al: High prevalence of human papillomaviruses in fresh frozen breast cancer samples. J Med Virol 83: 2157-2163, 2011.

2. Jones DL, Thompson DA and Munger K: Destabilization of the RB tumor suppressor protein and stabilization of p53 contribute to HPV type 16 E7-induced apoptosis. Virology 239: 97-107, 1997.

3. Honda R and Yasuda H: Activity of MDM2, a ubiquitin Ligase, toward p53 or itself is dependent on the RING finger domain of the ligase. Oncogene 19: 1473-1476, 2000.

4. DiMaio D: The role of human papillomaviruses in cancer. J Neurovirol 14: 50-50, 2005.

5. Liu YM, McKalip A and Herman B: Human papillomavirus type 16 E6 and HPV-16 E6/E7 sensitize human keratinocytes to apoptosis induced by chemotherapeutic agents: Roles of p53 and caspase activation. J Cell Biochem 78: 334-349, 2000.

6. Meek DW: The p53 response to DNA damage. DNA Repair 3: 1049-1056, 2004.

7. Chang YC, Liao CB, Hsieh PY, Liou ML and Liu YC: Expression of tumor suppressor p53 facilitates DNA repair but not UV-induced G2/M arrest or apoptosis in Chinese hamster ovary CHO-K1 cells. J Cell Biochem 103: 528-537, 2008.

8. Wei J, O'Brien D, Vilgelm A, et al: Interaction of Helicobacter pylori with gastric epithelial cells is mediated by the p53 protein family. Gastroenterology 134: 1412-1423, 2008.

9. Mayer C and Grummt I: Cellular stress and nucleolar function. Cell Cycle 4: 1036-1038, 2005.

10. Qian H, Wang T, Naumovski L, Lopez CD and Brachmann RK: Groups of $\mathrm{p} 53$ target genes involved in specific p53 downstream effects cluster into different classes of DNA binding sites. Oncogene 21: 7901-7911, 2002.

11. Sherman L and Schlegel R: Serum- and calcium-induced differentiation of human keratinocytes is inhibited by the E6 oncoprotein of human papillomavirus type 16. J Virol 70 : 3269-3279, 1996.

12. Liang XH, Volkmann M, Klein R, Herman B and Lockett SJ: Co-localization of the tumor-suppressor protein p53 and human papillomavirus E6 protein in human cervical carcinoma cell lines. Oncogene 8: 2645-2652, 1993.

13. Kelley ML, Keiger KE, Lee CJ and Huibregtse JM: The global transcriptional effects of the human papillomavirus E6 protein in cervical carcinoma cell lines are mediated by the E6AP ubiquitin ligase. J Virol 79: 3737-3747, 2005.

14. Tao M, Kruhlak M, Xia S, Androphy E and Zheng ZM: Signals that dictate nuclear localization of human papillomavirus type 16 oncoprotein E6 in living cells. J Virol 77: 13232-13247, 2003.

15. Li X and Coffino P: High-risk human papillomavirus E6 protein has two distinct binding sites within p53, of which only one determines degradation. J Virol 70: 4509-4516, 1996.

16. Momand J and Zambetti GP: Mdm-2: ‘big brother' of p53. J Cell Biochem 64: 343-352, 1997.
17. Butz K, Shahabeddin L, Geisen C, Spitkovsky D, Ullmann A and Hoppe-Seyler F: Functional p53 protein in human papillomaviruspositive cancer cells. Oncogene 10: 927-936, 1995.

18. Chalfie M, Tu Y, Euskirchen G, Ward WW and Prasher DC: Green fluorescent protein as a marker for gene expression. Science 263: 802-805, 1994.

19. Zheng Y, Zhang J and Rao Z: Ribozyme targeting HPV16 E6E7 transcripts in cervical cancer cells suppresses cell growth and sensitizes cells to chemotherapy and radiotherapy. Cancer Biol Ther 3: 1129-1135, 2004.

20. Gross-Mesilaty S, Reinstein E, Bercovich B, et al: Basal and human papillomavirus E6 oncoprotein-induced degradation of Myc proteins by the ubiquitin pathway. Proc Natl Acad Sci USA 95: 8058-8063, 1998.

21. Ronco LV, Karpova AY, Vidal M and Howley PM: Human papillomavirus $16 \mathrm{E} 6$ oncoprotein binds to interferon regulatory factor-3 and inhibits its transcriptional activity. Genes Dev 12: 2061-2072, 1998

22. Kumar A, Zhao Y, Meng G, et al: Human papillomavirus oncoprotein E6 inactivates the transcriptional coactivator human ADA3. Mol Cell Biol 22: 5801-5812, 2002.

23. Ferrigno $P$ and Silver PA: Regulated nuclear localization of stress-responsive factors: how the nuclear trafficking of protein kinases and transcription factors contributes to cell survival. Oncogene 18: 6129-6134, 1999.

24. Hollstein M, Rice K, Greenblatt MS, et al: Database of p53 gene somatic mutations in human tumors and cell lines. Nucleic Acids Res 22: 3551-3555, 1994.

25. Diaz D, Santander MA and Chavez JA: HPV-16 E6 and E7 oncogene expression is downregulated as a result of Mdm2 knockdown. Int J Oncol 41: 141-146, 2012.

26. Hwang SJ, Suh MJ, Yoon JH, et al: Identification of characteristic molecular signature of Mullerian inhibiting substance in human HPV-related cervical cancer cells. Int J Oncol 39: 811-820, 2011

27. Kawamata Y, Mitsuhashi A, Unno Y, et al: HPV 16-E6-mediated degradation of intrinsic p53 is compensated by upregulation of p53 gene expression in normal cervical keratinocytes. Int J Oncol 21: 561-567, 2002.

28. Fouret P, Dabit D, Sibony M, et al: Expression of p53 protein related to the presence of human papillomavirus infection in precancer lesions of the larynx. Am J Pathol 146: 599-604, 1995.

29. Isaacs JS, Chen P, Garza A, Hansen MF, Barrett JC and Weissman BE: Failure of HPV E6 to rapidly degrade p53 in human HeLa x PNET cell hybrids. Oncogene 14: 1669-1678, 1997.

30. Shin YC, Nakamura H, Liang X, et al: Inhibition of the ATM/ p53 signal transduction pathway by Kaposi's sarcoma-associated herpesvirus interferon regulatory factor 1. J Virol 80: 2257-2266, 2006.

31. Accardi R, Dong W, Smet A, et al: Skin human papillomavirus type 38 alters p53 functions by accumulation of deltaNp73. EMBO Rep 7: 334-340, 2006.

32. Li L, Guo L, Tao Y, et al: Latent membrane protein 1 of EpsteinBarr virus regulates p53 phosphorylation through MAP kinases. Cancer Lett 255: 219-231, 2007.

33. Granja AG, Nogal ML, Hurtado C, et al: Modulation of p53 cellular function and cell death by African swine fever virus. J Virol 78: 7165-7174, 2004. 